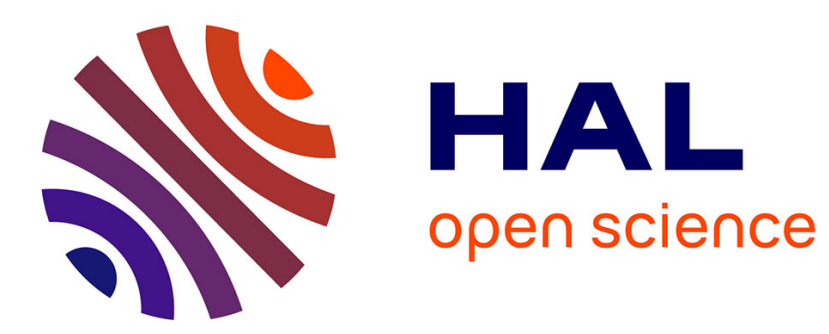

\title{
Effects of the angle of impact on the aeroacoustic feedback mechanism in supersonic impinging planar jets Christophe Bogey, Romain Gojon
}

\section{To cite this version:}

Christophe Bogey, Romain Gojon. Effects of the angle of impact on the aeroacoustic feedback mechanism in supersonic impinging planar jets. International Journal of Aeroacoustics, 2019, 18, pp.258 278. 10.1177/1475472X18812808. hal-02333806

\section{HAL Id: hal-02333806 https://hal.science/hal-02333806}

Submitted on 25 Oct 2019

HAL is a multi-disciplinary open access archive for the deposit and dissemination of scientific research documents, whether they are published or not. The documents may come from teaching and research institutions in France or abroad, or from public or private research centers.
L'archive ouverte pluridisciplinaire HAL, est destinée au dépôt et à la diffusion de documents scientifiques de niveau recherche, publiés ou non, émanant des établissements d'enseignement et de recherche français ou étrangers, des laboratoires publics ou privés. 


\title{
Effects of the angle of impact on the aeroacoustic feedback mechanism in supersonic impinging planar jets
}

International Journal of Aeroacoustics 2019, Vol. 18(2-3) 258-278

(C) The Author(s) 2018

Article reuse guidelines: sagepub.com/journals-permissions DOI: $10.1|77 /| 475472 \times 188 \mid 2808$ journals.sagepub.com/home/jae

\section{Romain Gojon' and Christophe Bogey ${ }^{2}$}

\begin{abstract}
Three planar impinging supersonic jets of infinite extent are simulated using compressible large eddy simulations in order to study the effects of the angle of impact on the flow and acoustic fields of the jet. At the exit of a nozzle of height $h$, they are ideally expanded and have an exit velocity $u_{j}$, yielding a Mach number of 1.28 and a Reynolds number of $5 \times 10^{4}$. They impinge on a flat plate at a distance $5.5 \mathrm{~h}$ from the nozzle lips with angles of $60^{\circ}, 75^{\circ}$, and $90^{\circ}$ between the jet direction and the plate. Mean velocity flows and snapshots of density, pressure, and vorticity are first shown. The mean convection velocity of the turbulent structures in the jet shear layers is then determined. The sound pressure levels are computed, and several tones due to the presence of a feedback mechanism are found to establish between the nozzle lips and the flat plate. They agree well with the corresponding measurements and with the classical model of the feedback mechanism. Moreover, when the angle of impact deviates from $90^{\circ}$ to $75^{\circ}$, a jump from the third to the fourth mode of the feedback mechanism and a reduction in intensity are noted. By applying a Fourier decomposition to the near pressure fields, hydrodynamic-acoustic standing waves are found for each dominant tone frequency. Moreover, as suggested by amplitude fields and velocity spectra in the jet shear layers, the feedback mechanism seems to establish mainly along the lip that is farther away from the plate when the impact angle is not normal. This jump from the third to the fourth mode is similar to the jump observed experimentally for an angle of impact of $90^{\circ}$ when the nozzle-to-plate distance increases from 5.5h to 5.85h. Finally, for an angle of impact of $60^{\circ}$, it is seen that none of the modes of the feedback persists in time, but that several modes randomly establish during short periods of time. These rapid switches
\end{abstract}

\footnotetext{
'ISAE-SUPAERO, Université de Toulouse, Toulouse, France

${ }^{2}$ Laboratoire de Mécanique des Fluides et d'Acoustique, UMR CNRS 5506, Ecole Centrale de Lyon, Université de Lyon, Lyon, Ecully, France
}

Corresponding author:

Romain Gojon, ISAE-SUPAERO, Université de Toulouse, 10 Avenue Edouard Belin, 31400 Toulouse, France.

Email: romain.gojon@isae-supaero.fr 
between different modes lead to several tones that are less energetic on average and centered on $S t=0.25$.

\section{Keywords}

Impingement tones, large eddy simulation, supersonic flow

Date received: 5 March 2018; accepted: 2 July 2018

\section{Introduction}

In the acoustic field of high-subsonic and supersonic impinging jets, intense acoustic tones have been observed by many authors. Powell ${ }^{1}$ suggested, in 1953, that these tones are due to a feedback mechanism establishing between the nozzle lips and the plate. This mechanism consists of two steps. First, in the shear layer, a turbulent structure is convected downstream from the nozzle to the plate. The structure impinges on the plate and generates an acoustic wave propagating upstream towards the nozzle. This wave is then reflected by the nozzle lip, excites the shear layer, and leads to the formation of a new turbulent structure.

Round subsonic and supersonic jets impinging normally on a flat plate have been studied by numerous researchers. Subsonic jets were notably studied by Ho and Nosseir ${ }^{2}$ and Nosseir and $\mathrm{Ho}^{3}$ who always observed intense acoustic tones for exit Mach numbers ranging from 0.3 to 0.9 . Supersonic jets were analyzed by Henderson and Powell, ${ }^{4}$ Krothapalli et al., ${ }^{5}$ Risborg and Soria, ${ }^{6}$ Buchmann et al., ${ }^{7}$ and Weightman et al. ${ }^{8}$ A feedback mechanism similar to those in subsonic jets was found. This mechanism appears very often when the jet is ideally expanded but only for some nozzle-to-plate distances when the jet is imperfectly expanded. ${ }^{9}$ More recently, the authors studied in depth the tone production mechanisms in underexpanded and ideally expanded impinging round jet using compressible large eddy simulation (LES). ${ }^{10-12}$ Planar jets impinging on a flat plate normally also produce intense tone frequencies. However, compared to round jets, Arthurs and Ziada ${ }^{13}$ noted that tones are visible at lower flow velocities. Planar supersonic jets impinging on a flat plate normally have been studied notably by Norum ${ }^{14}$ and Tam and Norum ${ }^{15}$ in the 1990s. Often, two emerging tones were observed in the acoustic spectra of the jets. The lower tone frequency was found to be associated with a symmetric oscillation mode of the jet and the upper one, with an antisymmetric oscillation mode of the jet. Using a simple two-dimensional (2D) simulation by Hourigan et al. ${ }^{16}$ was able to recover these tones. To explain this phenomenon, Tam and Norum ${ }^{15}$ proposed that the upstream propagating waves of the feedback mechanism are neutral acoustic wave modes of the jet. Using a vortex sheet jet model, allowable frequency ranges were found for upstream propagating neutral acoustic wave mode of the jet, depending on their symmetric or antisymmetric nature. These allowable ranges were in agreement with the tones observed in the experiment of Norum. ${ }^{14}$ This model has been used by the authors in combination with the classical aeroacoustic feedback model in order to predict, for a given ideally expanded planar impinging jet, the most likely tone frequency and the associated jet oscillation. ${ }^{17} \mathrm{~A}$ generalization to the case of ideally expanded round impinging jets has later been proposed in Bogey and Gojon. ${ }^{11}$ In this paper, using a space-time Fourier transform on the fluctuating pressure inside the jet, upstream propagating waves with a group velocity and a phase velocity very close to the ambient speed of 
sound have been observed. They correspond to neutral acoustic wave modes of the jet and can be found using a vortex sheet model. Recently, ${ }^{18}$ the same decomposition has been applied to screeching jets. It is shown that the feedback loop, responsible for dominant modes $A$ and $C$ and secondary mode $u$, is closed by acoustic upstream propagating waves in the jet which belongs to the neutral acoustic wave modes of the equivalent ideally expanded jet.

For non-normal impingement angles, the intensity of the feedback mechanism decreases because of the loss of symmetry. For instance, for a supersonic ideally expanded planar jet impinging on an inclined flat plate, Norum ${ }^{14}$ noted experimentally that the amplitude of the strongest impingement tone decreases when the angle of impact deviates from $90^{\circ}$. In order to understand the lift-off phase of a space launcher, Nonomura et al. ${ }^{19,20}$ performed the simulation of a 2D jet impinging on a inclined plate and the LES of a three dimensional (3D) supersonic ideally expanded round jet impinging on a inclined plate. They obtained three types of waves in the acoustic field, namely, the Mach waves generated in the jet shear layers, the Mach waves from the shear layer of the supersonic flow on the inclined flat plate, and the waves coming from the impingement region. These three types of waves were analyzed by Tsutsumi et al. ${ }^{21}$ using a snapshot proper orthogonal decomposition combined with Fourier transform.

In the present work, the LESs of three planar supersonic jets of infinite extent are carried out for different angles of impact in order to investigate the feedback mechanism between the nozzle lips and the flat plate. This work follows a previous study ${ }^{17}$ in which planar supersonic jets impinging normally on a flat plate were simulated. More precisely, a case from the previous study for a normal impingement angle is considered, and two others are considered for angles of impact of $60^{\circ}$ and $75^{\circ}$. The paper is organized as follows. The main characteristics of the jets and the simulations are presented in the following section. Next, mean fields, snapshots, and fluctuating fields are shown and compared with experimental data. Furthermore, the acoustic results are then shown where the tone frequencies of the feedback mechanism are studied. Finally, concluding remarks are given.

\section{Parameters}

\section{Jets parameters}

Three planar jets impinging on an inclined flat plate are computed using compressible LES. The different cases referred to as JetL5-60d, JetL5-75d, and JetL5-90d are presented in Table 1: the three angles of impact of $\theta=60, \theta=75$, and $\theta=90^{\circ}$ between the jet axis, and the flat plates are considered. The case JetL5-90d was simulated in a previous study. ${ }^{17}$ In all cases, the nozzle-to-plate distance along the jet axis is equal to $L=5.5 h$, where $h$ is the height on the nozzle, whereas the nozzle-to-plate distance increases from $5.5 h$ to $5.79 h$ along the upper lip line at $y=h / 2$ and decreases from $5.5 h$ to $5.21 h$ along the lower lip line at $y=-h / 2$ when the angle of impact deviates from $90^{\circ}$ to $60^{\circ}$. The jets originate from a planar nozzle of height $h$ and width $l=3.25 h$ in the spanwise direction. The lip thickness is $e=0.5 h$. Periodic conditions are imposed in the spanwise direction, permitting to simulate a planar jet of infinite extent. The jets are ideally expanded and have a Mach number of $\mathcal{M}_{j}=u_{j} / a_{j}=1.28$ where $u_{j}$ is the jet exit velocity and $a_{j}$ is the speed of sound in the jet. Their Reynolds is $R e_{j}=u_{j} h / \nu=5 \times 10^{4}$, where $\nu$ is the kinematic molecular viscosity. 
Table I. Jet parameters: Mach number $\mathcal{M}_{j}=u_{j} / a_{j}$, Reynolds number $\operatorname{Re}_{j}=u_{j} h / \nu$, nozzle-to-plate distance $L$, angle $\theta$ between the jet direction, and the flat plate and nozzle-to-plate distances along the upper and the lower lip lines $L_{\text {upper }}$ and $L_{\text {lower }}$

\begin{tabular}{lllllll}
\hline & $\mathcal{M}_{j}$ & $R e_{j}$ & $L$ & $\theta$ & $L_{\text {upper }}$ & $L_{\text {lower }}$ \\
\hline JetL5-90d & 1.28 & $5 \times 10^{4}$ & $5.5 \mathrm{~h}$ & $90^{\circ}$ & $5.5 \mathrm{~h}$ & $5.5 \mathrm{~h}$ \\
JetL5-75d & 1.28 & $5 \times 10^{4}$ & $5.5 \mathrm{~h}$ & $75^{\circ}$ & $5.63 \mathrm{~h}$ & $5.37 \mathrm{~h}$ \\
JetL5-60d & 1.28 & $5 \times 10^{4}$ & $5.5 \mathrm{~h}$ & $60^{\circ}$ & $5.79 \mathrm{~h}$ & $5.21 \mathrm{~h}$ \\
\hline
\end{tabular}

The ejection conditions of the jets and the nozzle-to-plate distances are similar to the parameters in the experimental study of Thurow et al. ${ }^{22}$

\section{Numerical parameters}

For the case where the angle between the jet axis and the flat plate is $90^{\circ}$, the unsteady compressible Navier-Stokes equations are solved on a Cartesian mesh for which $x, y$, and $z$ denote the longitudinal, lateral, and spanwise directions, respectively. An explicit six-stage Runge-Kutta algorithm is used for time integration, and low-dispersion 11-point explicit finite differences are used for spatial derivation. ${ }^{23,24}$ At the end of each time step, a relaxation filtering is applied to the flow conservative variables in order to remove grid-to-grid oscillations and to dissipate subgrid-scale turbulent energy. The radiation conditions of Tam and Dong ${ }^{25}$ are implemented at the inflow and lateral boundaries of the computational domain. A sponge zone combining grid stretching and Laplacian filtering is also employed to damp the turbulent fluctuations before they reach the lateral boundaries. Adiabatic noslip conditions are imposed to the nozzle wall and the flat plate. Examples of 3D subsonic jets simulated using the present solver can be found in Bogey et al. ${ }^{26,27}$ A shock-capturing filtering is applied in order to avoid Gibbs oscillations near shocks. It consists in applying a conservative second-order filter at a magnitude determined each time step using a shock sensor. $^{28}$ This method was successfully used by de Cacqueray et al. ${ }^{29}$ for the LES of a nonideally expanded jet at an equivalent Mach number $\mathcal{M}_{j}=3.3$ and later by Gojon and Bogey $^{30}$ for a supersonic screeching jet with $\mathcal{M}_{j}=1.56$. Finally, at the nozzle exit, a Blasius boundary-layer velocity profile is imposed with a boundary-layer thickness of $0.075 h$, and vortical disturbances not correlated in the spanwise direction ${ }^{26}$ are added in the boundary layer in the nozzle at $x=-0.5 h$ in order to generate velocity fluctuations at the nozzle exit. The strength of the forcing is chosen in order to obtain peak turbulent intensities higher than $4 \%$ of the jet exit velocity $u_{j}$ at the nozzle exit. More precisely, they are equal to $7.3 \%, 5.2 \%$, and $4.3 \%$ of $u_{j}$ for JetL5-90d, JetL5-75d, and JetL5-60d, respectively. The jets are thus highly disturbed at the nozzle exit.

For the two cases where $\theta=60^{\circ}$ and $\theta=75^{\circ}$, two Cartesian meshes are used. The meshes as represented in Figure 1 for the JetL5-75d. The mesh containing the nozzle is referred to as the primary grid, and the mesh close to the flat plate as the secondary grid. The two meshes are identical in the spanwise direction $z$, allowing the flow variables to be transferred from one grid to another using a 2D interpolation in the $(x, y)$ plane. Optimized interpolation centered schemes for computational aeroacoustics were developed by $\mathrm{Tam}$ and $\mathrm{Hu}^{31}$ and by Chicheportiche and Gloerfelt, ${ }^{32}$ for instance. They are developed from Lagrangian polynomial, minimizing the error in the wave number space. However, for a centered 2D interpolation, the improvement relative to the Lagrangian $2 \mathrm{D}$ interpolation is weak. ${ }^{32}$ 


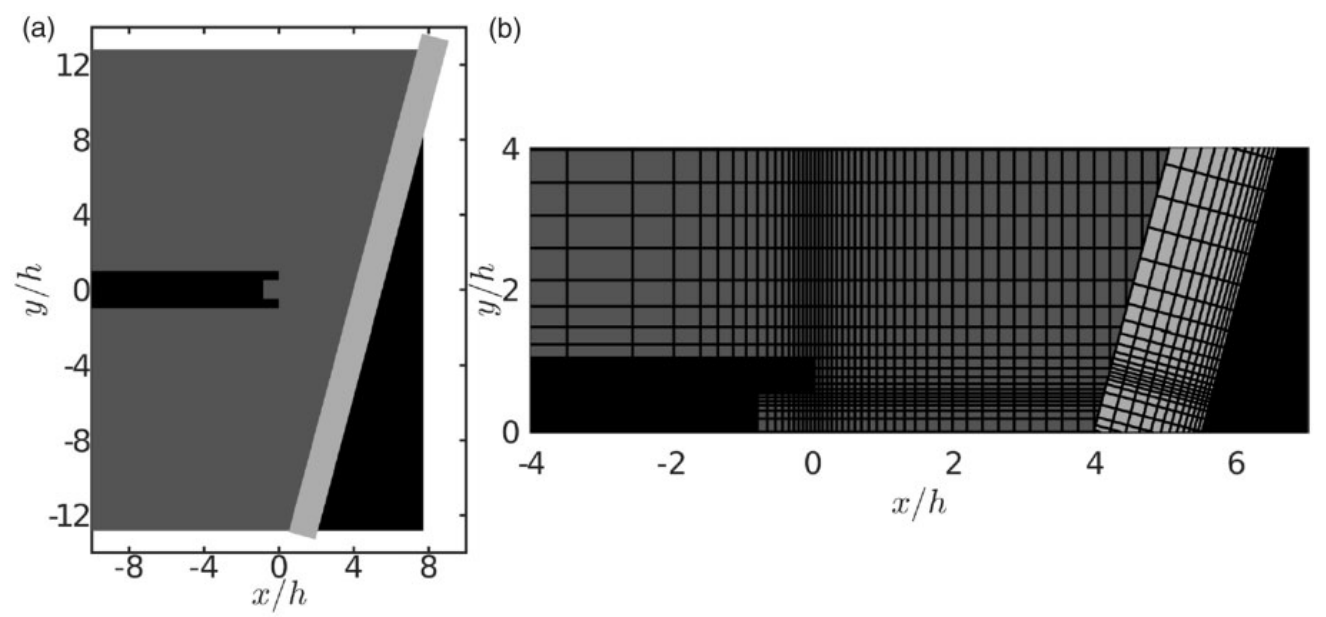

Figure I. Representation of the two Cartesian meshes for JetL5-75d; (a) sketch of the two meshes, with the primary grid in dark gray, the secondary grid in light gray, and the nozzle and flat plate in black and (b) representation of the grids; every 15 points is shown.

Thus, in the present work, a high-order Lagrangian 2D interpolation is used in each direction. A 10-point stencil is chosen for the interpolation as it is the best compromise between accuracy and computational time, as suggested by Marsden et al. ${ }^{33}$ and Chicheportiche and Gloerfelt. ${ }^{32}$ Therefore, the formal order of the interpolation is 10 . In practice, the value of the variable $u$ of the receiving point $\left(x_{l}, y_{l}\right)$ is computed from the $10 \times 10=100$ giving points $(x(i), y(j))$ as

$$
u\left(x_{l}, y_{l}\right)=\sum_{i, j=1}^{N} S_{i j} u(x(i), y(j))
$$

where the coefficients $S_{i j}$ are the $N^{2}$ interpolation coefficients. As reported earlier, the Lagrangian polynomials were chosen, giving

$$
S_{i j}=\prod_{k=1, k \neq i}^{N} \frac{x_{l}-x(i)}{x(k)-x(i)} \prod_{l=1, l \neq j}^{N} \frac{y_{l}-y(j)}{y(l)-y(j)}
$$

The simulations are carried out using an OpenMP-based in-house solver, and a total of 200,000 iterations are made in each case after the transitional period. The simulation time is equal to $500 \mathrm{~h} / u_{j}$. The Cartesian meshes contain between 191 and 210 million points for the primary grids and between 38 and 46 million points for the secondary grids, as noted in Tables 2 and 3, respectively.

The variations of the axial and lateral mesh spacings in the grid used for JetL5-90d are presented in a previous paper. ${ }^{17}$ The minimal axial mesh spacing, near the nozzle lips and the flat plate, is equal to $\Delta x=0.00375 h$, and the maximal axial mesh spacing, in the jet, is $\Delta x=0.015 h$. The lateral mesh spacings are equal to $\Delta y=0.00375 h$ at $y= \pm h / 2$ and to 
Table 2. Parameters of the primary grids containing the jets.

\begin{tabular}{lllll}
\hline & $n_{x}$ & $n_{y}$ & $n_{z}$ & Number of points \\
\hline JetL5-90d & 903 & 1051 & 219 & $208 \times 10^{6}$ \\
JetL5-75d & 966 & 901 & 219 & $191 \times 10^{6}$ \\
JetL5-60d & 1196 & 801 & 219 & $210 \times 10^{6}$ \\
\hline
\end{tabular}

Table 3. Parameters of the secondary grids close to the plate.

\begin{tabular}{lllll}
\hline & $n_{x}$ & $n_{y}$ & $n_{z}$ & Number of points \\
\hline JetL5-75d & 181 & 961 & 219 & $38 \times 10^{6}$ \\
JetL5-60d & 181 & 1151 & 219 & $46 \times 10^{6}$ \\
\hline
\end{tabular}

Table 4. Mesh spacings on the plate at $y=2 h$, in wall units.

\begin{tabular}{llll}
\hline & $\Delta x^{+}$ & $\Delta y^{+}$ & $\Delta z^{+}$ \\
\hline JetL5-90d & 10.1 & 55 & 40 \\
JetL5-75d & 13.2 & 68 & 47 \\
JetL5-60d & 14.7 & 70 & 49 \\
\hline
\end{tabular}

$\Delta y=0.03 h$ for $2.5 h \leq y \leq 8.5 h$. In the spanwise direction, the mesh spacing is equal to $\Delta z=0.015 \mathrm{~h}$. In the primary grids used for JetL5-60d and JetL5-75d, the minimal axial mesh spacing near the nozzle lip is equal to $\Delta x=0.00375 h$, and the maximal axial mesh spacing, downstream from the nozzle, is $\Delta x=0.015 \mathrm{~h}$. The lateral and spanwise mesh spacings are identical to those for JetL5-90d. For the secondary meshes, they have a minimal axial mesh spacing of $\Delta x=0.00375 h$ close to the plate and a maximal axial mesh spacing of $\Delta x=0.015 h$ upstream from the plate. Again, the lateral and spanwise mesh spacings are the same as those for JetL590d. They are chosen to minimize the difference in mesh size between the two grids as illustrated in Figure 1(b). Furthermore, the maximum mesh spacing of $0.03 \mathrm{~h}$ allows acoustic waves with Strouhal numbers up to $S t=f h / u_{j}=5.6$ to be well propagated, where $f$ is the frequency.

After the jet impact, wall jets develop on the flat plate. Their discretizations are discussed by considering the mesh spacings at the wall at $y=2 h$, given in Table 4 in wall units. In the four LES, values of about $\Delta y^{+}=65$ and $\Delta z^{+}=45$ are obtained in the streamwise and the spanwise directions, and $\Delta x^{+} \simeq 12$ is found in the wall-normal direction. Given that the mesh spacings necessary to compute turbulent wall-bounded flows using LES $^{34-37}$ are about of $\Delta^{+}=30$ in the streamwise direction, $\Delta^{+}=10$ in the spanwise direction, and $\Delta^{+}=1$ in the wall-normal direction, the meshes appear slightly too coarse to accurately compute the turbulent wall jets. However, this is not the main objective of the present simulations.

\section{Aerodynamic results}

\section{Flow snapshots}

Snapshots of the vorticity norm obtained in the $(x, y)$ plane for JetL5-90d, JetL5-75d, and JetL5-60d are represented in Figure 2. For the three cases, the two mixing layers exhibit 

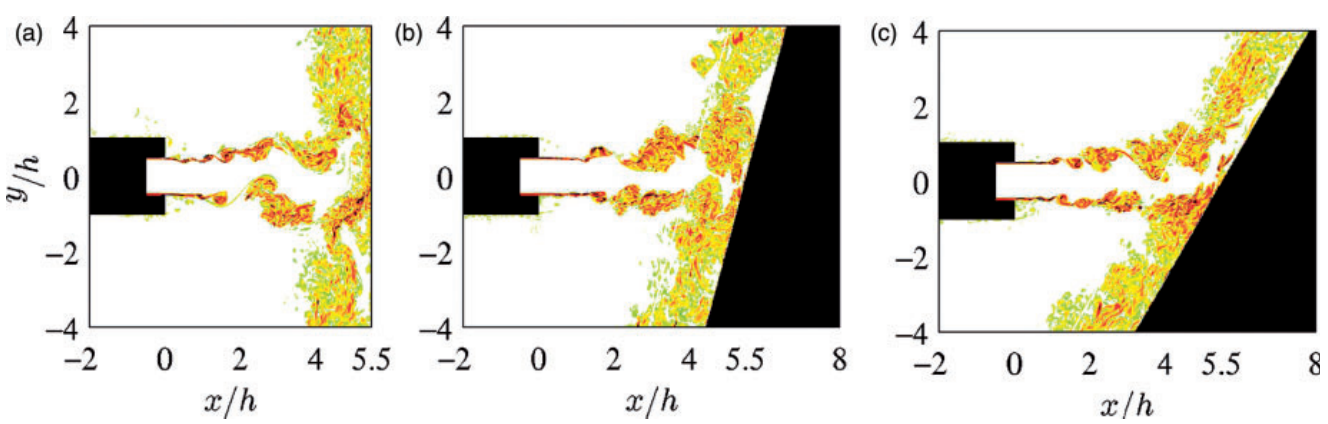

Figure 2. Snapshots obtained in the $(x, y)$ plane of vorticity norm $|\omega|=\sqrt{\omega_{x}^{2}+\omega_{y}^{2}+\omega_{z}^{2}}$ for (a) JetL5-90d, (b) JetL5-75d, and (c) JetL5-60d. The color scale ranges up to the level of $10 u_{j} / h$. The nozzle and the plate are in black.

large-scale structures, of typical size $0.5 h$. This result is similar to the experimental results provided by Thurow et al. ${ }^{22}$ for a rectangular supersonic jet impinging on a flat plate normally. Moreover, for JetL5-60d, the shear layers may not interact between each other near the region of impact, which seems to be the case for JetL5-90d and JetL5-75d.

In order to visualize the flow and acoustic fields of the jets, snapshots in the $(x, y)$ plane of the density and the fluctuating pressure are provided in Figure 3 and in a movie available online. In all cases, large-scale structures in the jet shear layers and upstream-propagating sound waves are observed. These waves appear to be generated in the region of jet impact and to have amplitudes decreasing significantly as the angle of impact deviates from $90^{\circ}$.

\section{Mean flow fields}

The mean total velocity fields obtained in the $(x, y)$ plane are shown in Figure 4. Very small variations of about $3 \%$ of the jet exit velocity are found near the jet axis, indicating that the jets are almost ideally expanded, as desired. In all cases, a stagnation point is visible on the flat plate at $y=0$, and plane wall jets form on the plate on both sides of the jet. The important scaling factors of plane wall jets are the maximum velocity $u_{m}$, the distance $x_{m}$ from the wall at which the velocity reaches $u_{m}$, and the distance $x_{1 / 2}$ from the wall at which the mean velocity has dropped to $u_{m} / 2 .{ }^{38,39}$ For the present jets, these factors are given in Table 5 along the lines $y= \pm 2 h$.

When the angle of impact is not normal, the developments of the top and bottom plane wall jets differ, as expected. For the top plane wall jets, the maximum velocity increases, but the length scales $x_{m}$ and $x_{1 / 2}$ remain nearly constant. Shock cells are also visible in these jets in Figure 4(b) and (c). For the bottom plane wall jets, the maximum velocity and the length scales $x_{m}$ and $x_{1 / 2}$ all decrease significantly when the angle of impact deviates from $90^{\circ}$.

\section{Convection velocity}

In order to describe the feedback mechanism, the convection velocity $u_{c}$ of the turbulent structures along the lip lines $y=h / 2$ and $y=-h / 2$ is calculated from cross-correlations of axial velocity fluctuations $u_{x}{ }^{\prime}$. The results are shown in Figure 5 for the three simulated jets. 
(a)

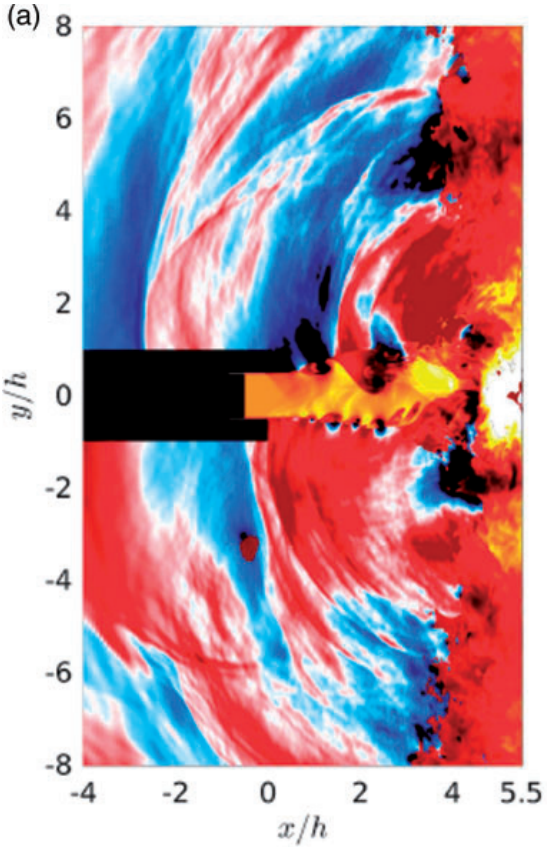

(b) 9

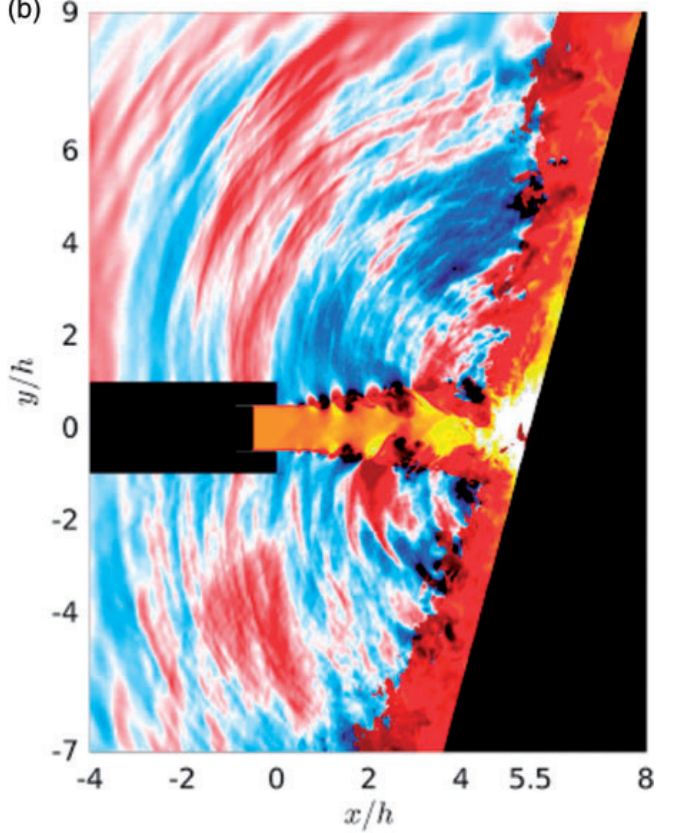

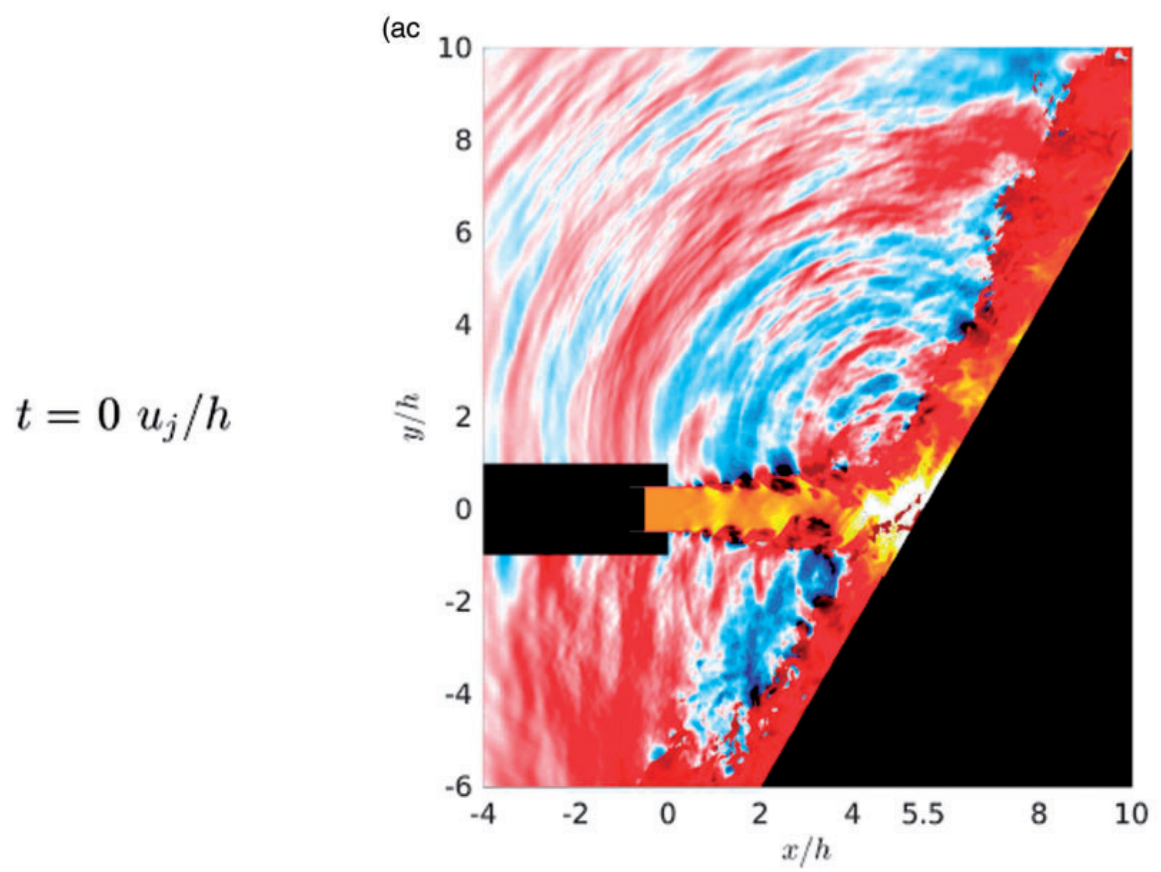

Figure 3. Snapshots obtained in the $(x, y)$ plane of density in the jet axis and close to the flat plate and of pressure fluctuations for (a) JetL5-90d, (b) JetL5-75d, and (c) JetL5-60d. The color scale ranges from I to $2 \mathrm{~kg} \cdot \mathrm{m}^{-3}$ for density and from -7500 to $7500 \mathrm{~Pa}$ for fluctuating pressure. The nozzle and the plate are in black. 

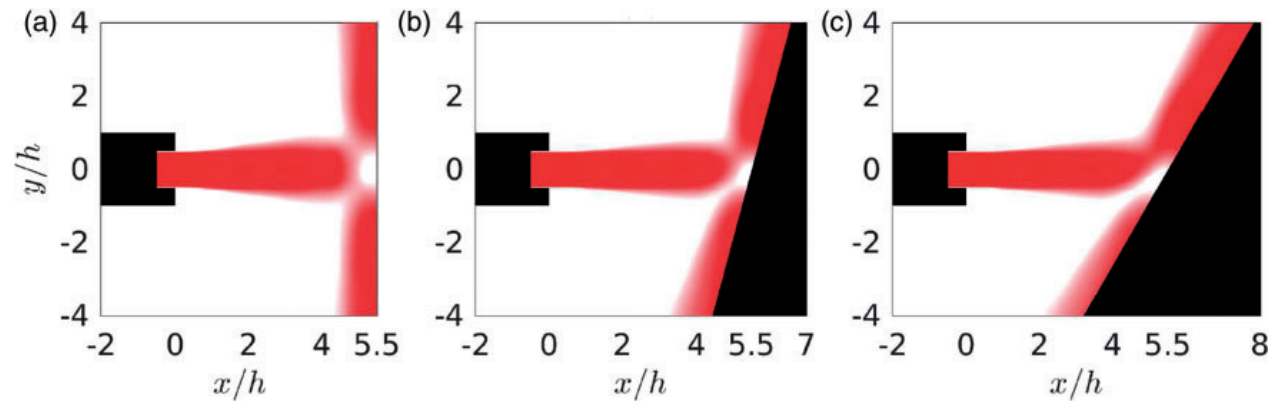

Figure 4. Mean velocity field obtained in the (x,y) plane for (a) JetL5-90d, (b) JetL5-75d, and (c) JetL5-60d. The color scale ranges from 0 to $400 \mathrm{~m} \cdot \mathrm{s}^{-1}$. The nozzle and the flat plate are in black.

Table 5. Maximum velocity $u_{m}$ and distances $x_{m} / h$ and $x_{1 / 2} / h$ of the wall jet at $y= \pm 2 h$.

\begin{tabular}{llllllll}
\hline & $y=2 h$ & & & & $y=-2 h$ & & \\
\cline { 2 - 3 } & $u_{m}\left(\mathrm{~m} \cdot \mathrm{s}^{-1}\right)$ & $x_{m} / h$ & $x_{1 / 2} / h$ & & $u_{m}\left(\mathrm{~m} \cdot \mathrm{s}^{-1}\right)$ & $x_{m} / h$ & $x_{1 / 2} / h$ \\
\hline JetL5-90d & 349 & 0.051 & 0.83 & & 349 & 0.051 & 0.83 \\
JetL5-75d & 375 & 0.045 & 0.90 & & 331 & 0.030 & 0.68 \\
JetL5-60d & 382 & 0.054 & 0.86 & & 284 & 0.012 & 0.53 \\
\hline
\end{tabular}

(a)

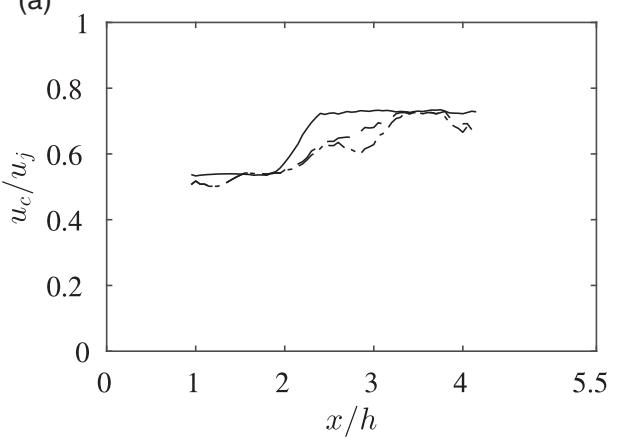

(b)

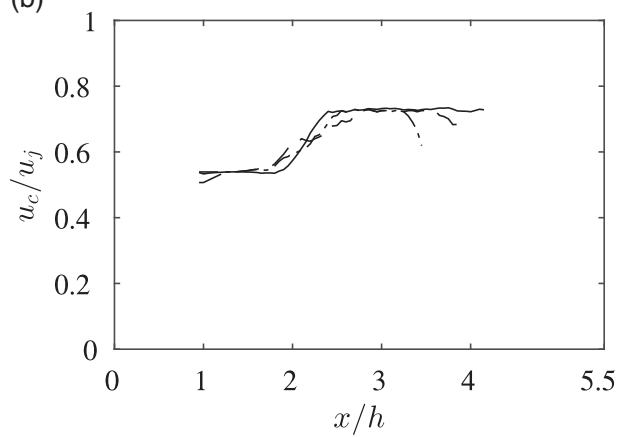

Figure 5. Convection velocity of the turbulent structures as a function of the axial coordinate in (a) the upper jet shear layer at $y=h / 2$ and (b) the lower jet shear layer at $y=-h / 2$ for — JetL5-90d, - - - JetL575d, and -.- JetL5-60d.

For all jets, the convection velocities are approximately of $0.45 u_{j}$ at $x=h$. Farther downstream, for JetL5-90d, the convection velocity increases and reaches a maximal value of $0.63 u_{j}$ at $x=2.5 h .{ }^{17}$ For JetL5-75d and JetL5-60d, similar behaviors are found along the lower jet shear layer, where maximal values of $0.63 u_{j}$ at $x=3.75 \mathrm{~h}$ are noted. However, along the upper jet shear layer, the increase in the convection velocity is slower, and maximal values of $0.63 u_{j}$ are also reached but further downstream, at $x=3.35 \mathrm{~h}$. Overall, the mean convection velocity of the large-scale structures along the lip line is of $\left\langle u_{c}\right\rangle \simeq 0.55 u_{j}$. This result is in agreement with the experimental results of Panda et al. ${ }^{40}$ who measured 
a mean convection velocity of $0.60 u_{j}$ for an ideally expanded rectangular supersonic jet with an exit Mach number of $\mathcal{M}_{e}=1.3$. Moreover, for two pressure-matched parallel streams with equal specific heats, the convection Mach number $\mathcal{M}_{c}$ was defined by Papamoschou and Roshko ${ }^{41}$ as

$$
\mathcal{M}_{c}=\frac{u_{1}-u_{2}}{a_{1}+a_{2}}=\frac{u_{1}-u_{c}}{a_{1}}=\frac{u_{c}-u_{2}}{a_{2}}
$$

where $u_{1}$ and $u_{2}$ are the high-speed and low-speed free-stream velocities, $a_{1}$ and $a_{2}$ are the speeds of sound, and $u_{c}$ is the theoretical isentropic convection velocity. For the present planar jets, $u_{1}$ is the jet velocity at the nozzle exit and $u_{2}$ is the velocity outside the jet, hence $u_{1}=u_{j}$ and $u_{2}=0$, and $a_{1}=a_{j}$ and $a_{2}=a_{0}$. Thus, the theoretical convection velocity for the planar jet is

$$
<u_{c}>=\frac{u_{j}}{a_{j} / a_{0}+1}
$$

yielding $\left\langle u_{c}\right\rangle=0.57 u_{j}$, in agreement with the simulation results.

\section{Overall sound pressure levels}

The overall sound pressure levels (OASPLs) obtained in the plane $(x, y)$ are represented for the three jets in Figure 6. Isocontours are added in order to improve the readability. The OASPLs, in $\mathrm{dB}$, are computed from the rms fluctuating pressure fields as

$$
\mathrm{OASPL}=10 \log \left(\frac{p_{r m s}^{\prime 2}}{p_{r e f}^{2}}\right)
$$

where $p_{\text {ref }}=2 \cdot 10^{-5} \mathrm{~Pa}$.

For JetL5-90d, in Figure 6(a), two acoustic components emerge. Let $\alpha$ be the angle at the impingement region between the upstream direction and the waves propagating from the flat
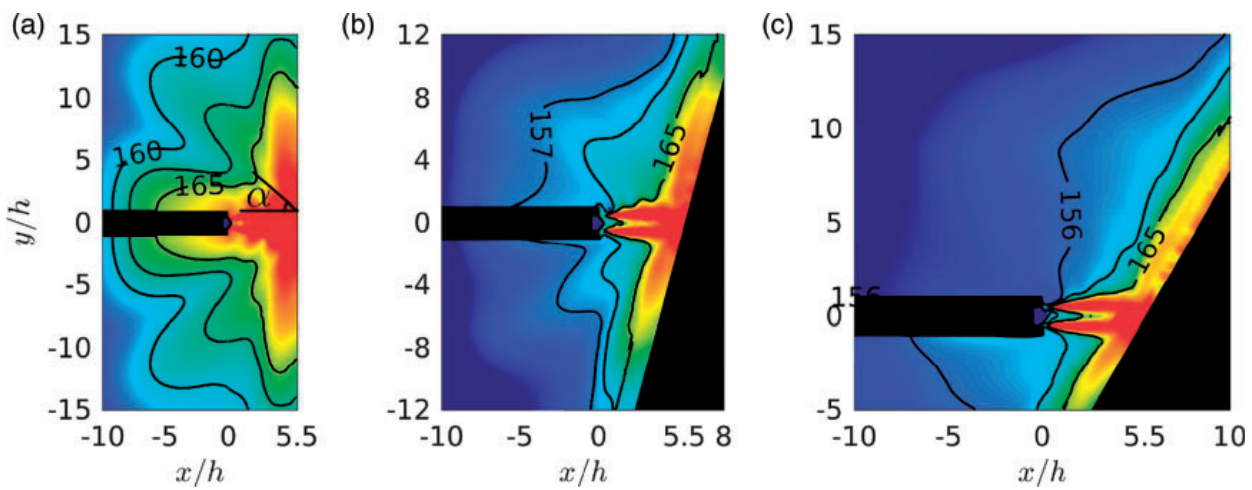

Figure 6. OASPL obtained in the $(x, y)$ plane for (a) JetL5-90d, (b) JetL5-75d, and (c) JetL5-60d. The color scale ranges from 150 to $175 \mathrm{~dB}$. The nozzle and the plate are in black. 
plate, as illustrated in Figure 6(a). The first acoustic component can be seen for $0^{\circ} \leq \alpha \leq$ $30^{\circ}$ and is therefore due to acoustic waves propagating in the upstream direction, closing the aeroacoustic feedback loop. The second acoustic contribution appears for $\alpha \simeq 50^{\circ}$ and seems to come from a region located near the plate, at $y \simeq 3.5 h$. For JetL5-75d, in Figure 6(b), the same two acoustic components are visible. The second acoustic component is here characterized by an angle of $\alpha \simeq 35^{\circ}$ in the upper side and $\alpha \simeq 30^{\circ}$ in the lower side. For JetL5-60d, in Figure 6(c), the acoustic radiation does not have a marked directivity. However, the presence of a supersonic top plane wall jet apparently leads to an additional acoustic component in the upper side, as observed numerically by Nonomura et al. ${ }^{20}$ for a round ideally expanded supersonic jet impinging on a flat plate with an angle of $45^{\circ}$.

\section{Acoustic results}

\section{Sound pressure levels}

The sound pressure levels computed at $x=0$ and $y=1.5 \mathrm{~h}$ are shown in Figure 7 as a function of the Strouhal number. They are computed using the Welch method with
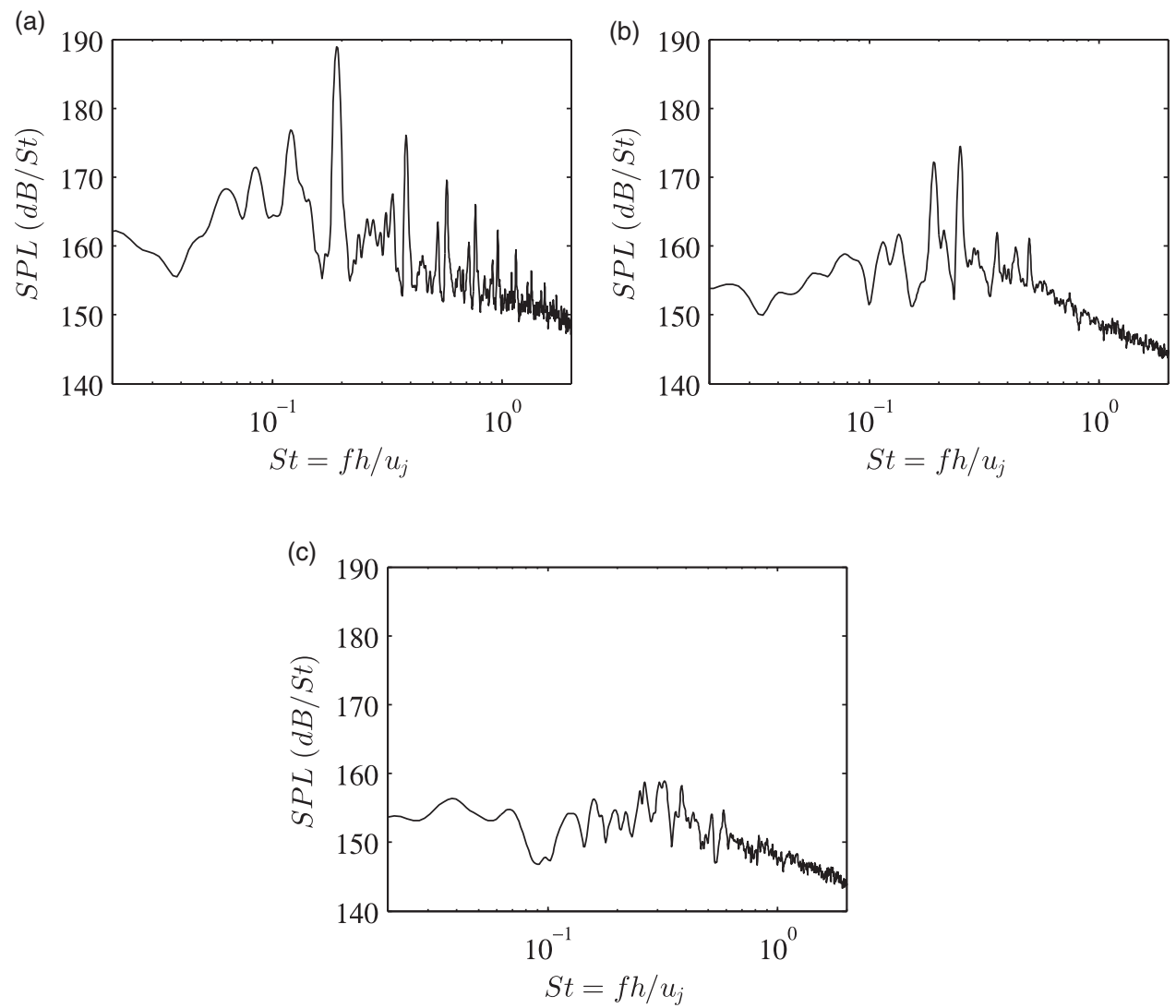

Figure 7. SPLs obtained at $x=0$ and $y=1.5 \mathrm{~h}$ as a function of the Strouhal number St for (a) JetL5-90d, (b) JetL5-75d, and (c) JetL5-60d. SPL: sound pressure level. 
Table 6. Tone Strouhal numbers in the spectra of Figure 7.

\begin{tabular}{llllllll}
\hline & $S t_{1}$ & $S t_{2}$ & $S t_{3}$ & $S_{4}$ & $S t_{5}$ & $S t_{6}$ & $S t_{7}$ \\
\hline JetL5-90d & 0.12 & $\mathbf{0 . 1 9}$ & 0.38 & 0.57 & 0.76 & 0.95 & 1.14 \\
JetL5-75d & 0.19 & $\mathbf{0 . 2 5}$ & - & - & - & - & - \\
JetL5-60d & $(0.25)$ & - & - & - & - & - & - \\
\hline
\end{tabular}

The Strouhal numbers of the dominant tones for JetL5-90d and JetL5-75d are in boldface. The Strouhal number at the maximum sound pressure level for JetL5-60d is between brackets.

a $50 \%$ overlapping in time and by performing spectral averaging over the periodic direction $z$. Several tone frequencies emerge and those whose levels are at least $10 \mathrm{~dB}$ higher than the broadband noise level are given in Table 6. For JetL5-90d, in Figure 7(a), a dominant tone frequency at $S t=0.19$ and its first six harmonics can be seen. Another tone frequency at $S t_{1}=0.12$ is visible. The agreement is excellent with the experimental data of Thurow et al. $^{22}$ who obtained a fundamental tone frequency at $S t=0.20$ for a similar jet. For JetL5-75d, in Figure 6(b), two emerging tone frequencies are found at $S t_{1}=0.19$ and $S t_{2}=0.25$. The first one is the same one as for the case JetL5-90d in Figure 7(a), but the latter one does not exist for JetL5-90d. Finally, for JetL5-60d, in Figure 7(c), there is no clear tone frequency. However, the maximum sound pressure level seems to be found around $S t=0.25$.

\section{Tone frequencies}

In order to explain the origin of tone frequencies, Powell ${ }^{1}$ suggested that a feedback mechanism establishes between the nozzle lips and the flat plate. Similarly, Ho and Nosseir ${ }^{2}$ and Nosseir and $\mathrm{Ho}^{3}$ constructed a simple model in order to predict the frequencies of the feedback mechanism, providing the relation

$$
\frac{N}{f}=\frac{L}{<u_{c}>}+\frac{L}{c_{0}}
$$

where $\left\langle u_{c}\right\rangle$ is the mean convection velocity of the large-scale structures in the shear layers and $N$ is the number of time the feedback mechanism occurs during one period of the feedback.

The Strouhal numbers of the tone frequencies obtained for the present jets in Figure 7 are plotted in Figure 8 as a function of the nozzle-to-plate distance $L / h$. For the three computed jets, the value $L=5.5 \mathrm{~h}$ at the center of the jet is used. Only the fundamental tone frequencies, which are not harmonics of other tone frequencies and which are called source tone frequencies in the following, are represented. The tone frequencies measured by Thurow et al., ${ }^{22}$ as well as those predicted using equation (6) with a mean convection velocity equal to $\left\langle u_{c}\right\rangle=0.55 u_{j}$, are also displayed. A good agreement is found between the simulation and the experimental data. Moreover, the tone frequencies seem to be well predicted by the model.

For JetL5-90d, where $L / h=5.5$, the two source tone frequencies at $S t_{1}=0.12$ and $S t_{2}=$ 0.19 can be associated with the second mode and the third mode of the feedback mechanism. For JetL5-75d, the two observable tones at $S t_{1}=0.19$ and $S t_{2}=0.25$ correspond to the 
Table 7. Maximal levels obtained in the sound spectra of Figure 7, skewness, and kurtosis factors of the fluctuating pressure at $x=0$ and $y=8.5 \mathrm{~h}$.

\begin{tabular}{llll}
\hline & JetL5-90d & JetL5-75d & JetL5-60d \\
\hline $\mathrm{SPL}_{\text {max }}\left(\mathrm{dB} / \mathrm{S}_{t}\right)$ & 188 & 175 & 160 \\
Skewness & 0.57 & 0.25 & 0.19 \\
Kurtosis & 3.87 & 3.2 & 3.49 \\
\hline
\end{tabular}

SPL: sound pressure level.

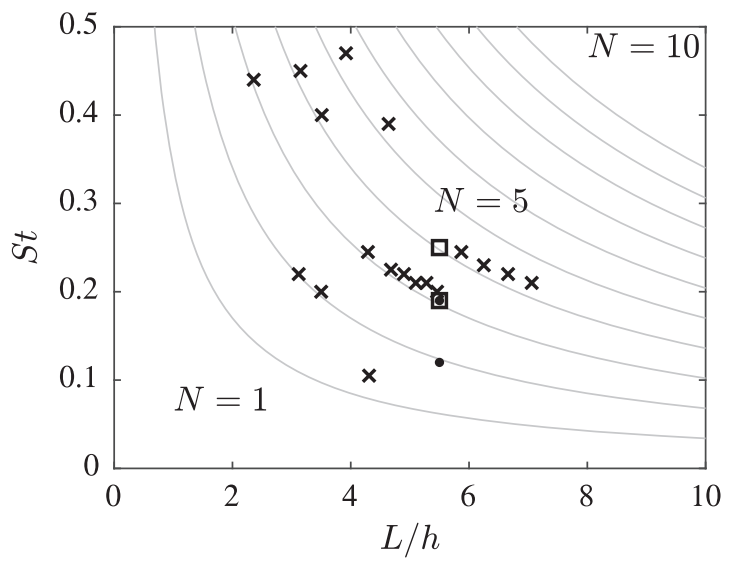

Figure 8. Strouhal numbers of the tone frequencies obtained - for JetL5-90d, $\square$ for JetL5-75d, and $\times$ experimentally by Thurow et al. ${ }^{22}$ as a function of the nozzle-to-plate distance. The gray lines show the values predicted by equation (6) using $\left\langle u_{c}\right\rangle=0.55 u_{j}$.

third and the fourth modes. Finally, for JetL5-60d, no tones are represented in Figure 8, as they do not stand out clearly in the spectra. The maximum sound pressure level is, however, reached around $S t=0.25$. Therefore, when the angle of impact deviates from $90^{\circ}$, there is a jump from the third mode to the fourth mode of the feedback mechanism. A similar jump is observed in the experiments of Thurow et al. ${ }^{22}$ when the nozzle-to-plate distance increases from $5.5 \mathrm{~h}$ to $5.85 \mathrm{~h}$. Indeed, as visible in Figure 8, they found dominant tone frequencies of $S t=0.20$ and $S t=0.245$ for $L=5.5 h$ and $L=5.85 h$, respectively.

\section{Skewness and kurtosis factors}

In order to investigate the non-linear features of the acoustic waves generated by the impingement, the statistical properties of the pressure are examined at $x=0$ and $y=8.5 h$. They are computed using the Welch method with a $50 \%$ overlapping in time and by performing statistical averaging over the periodic direction $z$. This point is located the furthest possible in the acoustic region where the mesh size is smaller than $0.03 h$. This position permits to resolve the acoustic spectrum up to a Strouhal numbers of $S t=f h / u_{j}=5.6$. It has been chosen in order to allow non-linear propagation effects to possibly affect the acoustic waves coming from the region of impact. Figure 9(a), (c), and (e) displays the fluctuating pressure at this point for the different jets. For JetL5-90d, in Figure 9(a), weak shock waves and $\mathrm{N}$-shaped waves showing sharp compressions associated with 

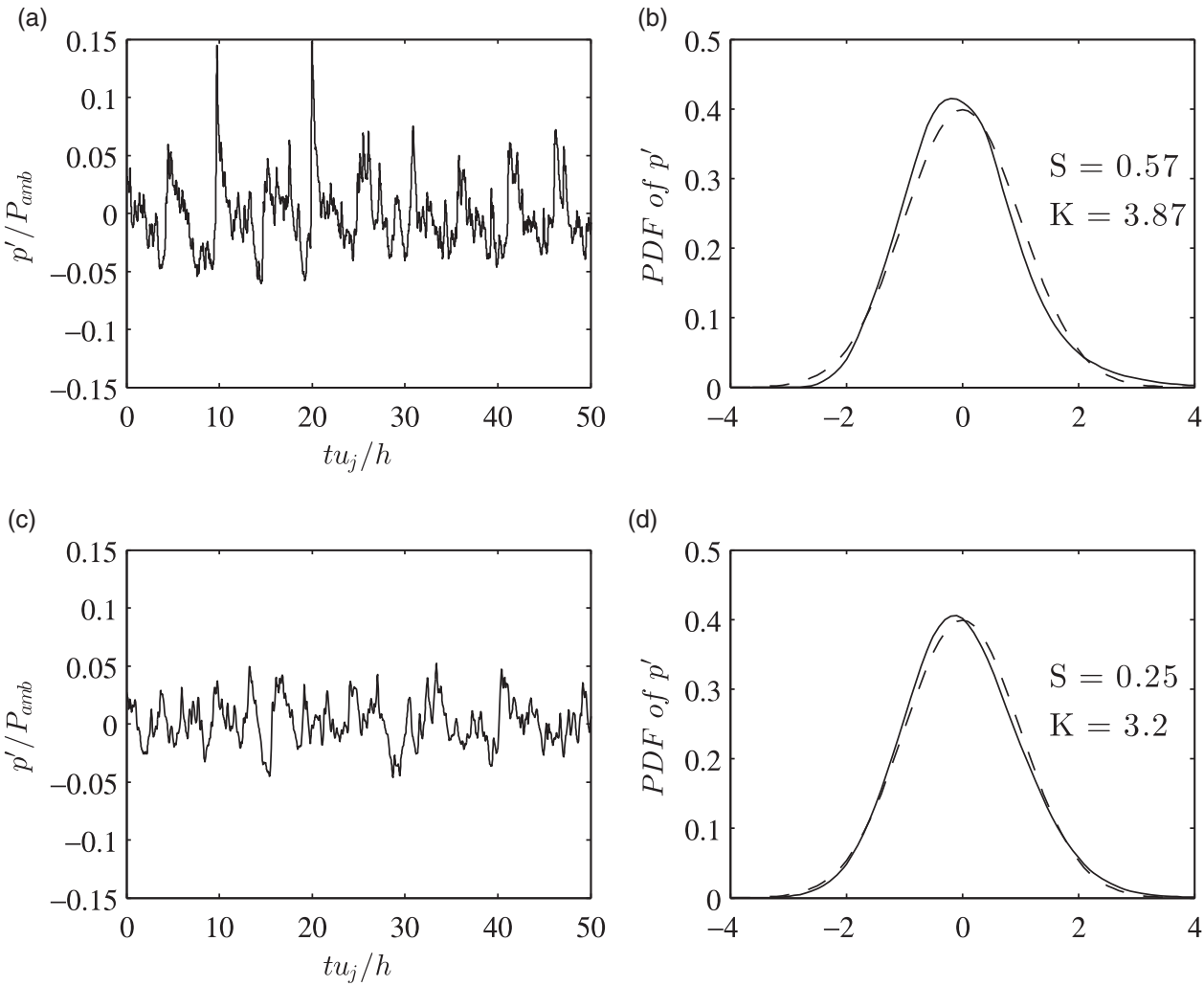

(d)

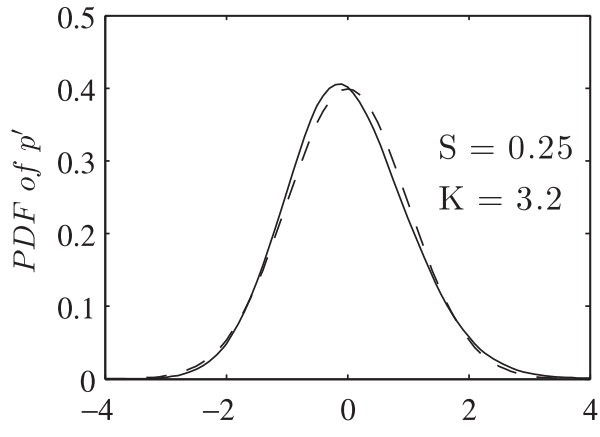

(e)

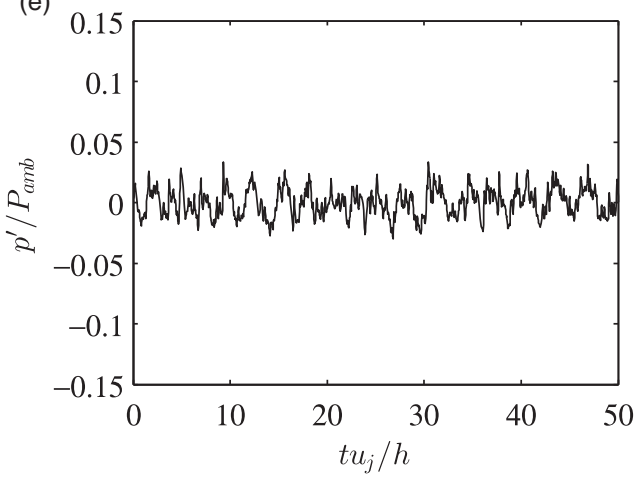

(f)

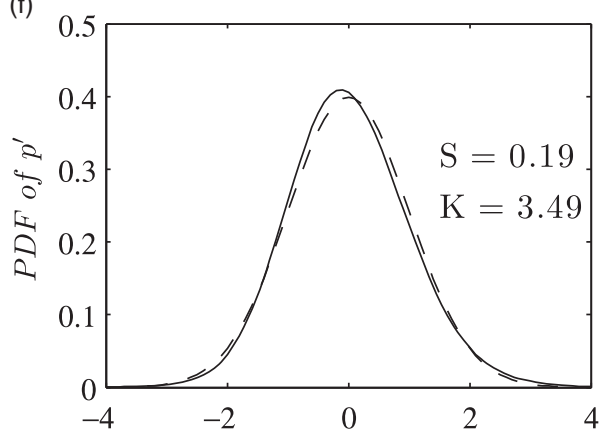

Figure 9. Left views: Fluctuating pressure at $x=0$ and $y=8.5 \mathrm{~h}$ over a short period, and right views: PDFs (- - PDF of a Gaussian distribution) for (a and b) JetL5-90d, (c and d) JetL5-75d, and (e and f) JetL560d. PDF: probability density function.

gradual expansions appear. These shapes are similar to those obtained by de Cacqueray and Bogey $^{42}$ in the acoustic field of an overexpanded jet ${ }^{29}$ at $\mathcal{M}_{j}=3.3$. Moreover, at some times, the fluctuating pressure exceeds $0.15 P_{a m b}$. This is the case at $t_{1}=10 \mathrm{~h} / \mathrm{u}_{j}$ and $t_{2}=20 \mathrm{~h} / \mathrm{u}_{j}$, where two strong shock waves are noticed. As the angle of impact changes, the amplitude of 
the pressure fluctuation decreases. N-shaped waves are still visible for JetL5-75d in Figure 9 (c) at $t_{1}=38 h / u_{j}$ but not for JetL5-60d in Figure 9(e).

In order to be more quantitative, Figure 9(b), (d), and (f) shows the probability density functions of the fluctuating pressure normalized by the standard deviation as well as the skewness and kurtosis factors for JetL5-90d, JetL5-75d, and JetL5-60d. For JetL5-90d, a skewness factor of $S=0.57$ and a kurtosis factor of $K=3.87$ are obtained, indicating strong non-linearity and intermittency of the signal. These properties correspond to those of the crackle noise first defined by Ffowcs-Williams et al. ${ }^{43}$ who identified this specific noise when the skewness factor of the pressure field exceeds 0.4. As the angle of impact deviates from $90^{\circ}$, the skewness factor decreases down to $S=0.25$ for JetL5-75d and to $S=0.19$ for JetpL5-60d. This result is in agreement with the weakening of the $\mathrm{N}$-shaped waves in the fluctuating pressure signals in Figure 9(c) and (e). Moreover, the kurtosis factor also decreases from $K=3.87$ for JetL5-90d down to $K=3.2$ and $K=3.49$ for JetL5-75d and JetL5-60d. This result indicates that the fluctuating pressure is not strongly intermittent for lower angles of impact.

For the three jets simulated, the peak values in the sound spectra at $\mathrm{x}=0$ and $\mathrm{y}=1.5 \mathrm{~h}$ of figure 7 , and the skewness and kurtosis factors of the fluctuating pressure at $\mathrm{x}=0$ and $y=8.5 \mathrm{~h}$, are collected in Table 7 . The maxima in the spectra and the skewness factors appear to be correlated. Moreover, the highest values of skewness and kurtosis factors are obtained for JetL5-90d, supporting that the most resonant case is also the case where non linearity and intermittency are the strongest.

\section{Fourier decomposition of the pressure field}

For each jet, the pressure field in one $(x, y)$ plane has been recorded every 50 th time step, at a sampling frequency of $S t=8$. A Fourier decomposition is then applied. In this way, for a given frequency, the amplitude and phase fields can be displayed.

The amplitude and phase fields obtained for the source tone frequency of JetL5-90d at $S t_{2}=0.19$, and the two tone frequencies at $S t_{1}=0.19$ and $S t_{2}=0.25$ of JetL5-75d are displayed in Figure 10. The amplitude fields are shown in the top views and the phase fields in the bottom views. The latter fields enable to identify the nature of the modes. The source tone frequencies of JetL5-90d and the secondary tone frequency of JetL5-75d at $S t_{1}=0.19$ can be associated to antisymmetric oscillation modes because there is a $180^{\circ}$ phase shift with respect to the jet axis in Figure 10(d) and (e). However, for the dominant source tone frequency of JetL5-75d at $S t_{2}=0.25$, a clear pattern of a symmetric or antisymmetric oscillation mode of the jet does not appear in Figure 10(c).

The amplitude fields of the three tone frequencies represented in the top views of Figure 10 all exhibit a cell structure between the jet nozzle and the plate. By considering the two semi-cells near the nozzle and the plate as one cell, the cell structures contain three cells for JetL5-90d at $S t_{2}=0.19$ and for JetL5-75d at $S t_{1}=0.19$ in Figure 10(a) and (b) and four cells for JetL5-75d at $S t_{1}=0.25$ in Figure 10(c). Thus, the number of cells corresponds to the mode number predicted by the model of $\mathrm{Ho}$ and Nosseir ${ }^{2}$ and Nosseir and $\mathrm{Ho}^{3}$ identified in Figure 8. Those cells are due to the formation of hydrodynamic-acoustic standing waves. Such waves have been observed experimentally by Panda et al. ${ }^{40}$ in supersonic screeching jets and numerically by Gojon et al. ${ }^{17}$ and Bogey and Gojon ${ }^{11}$ in ideally expanded planar impinging jets, among others. Moreover, for JetL5-75d, in the amplitude fields of Figure 10(b) and (c), higher amplitudes are found in the upper shear layer than in 


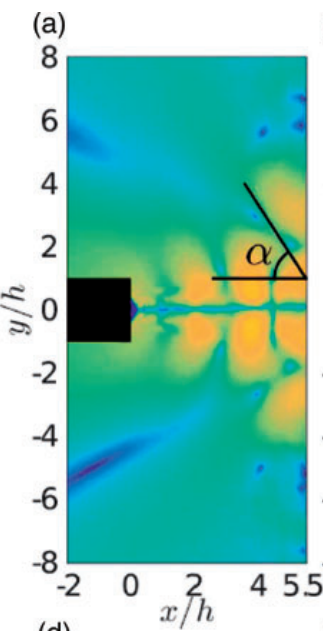

(b)
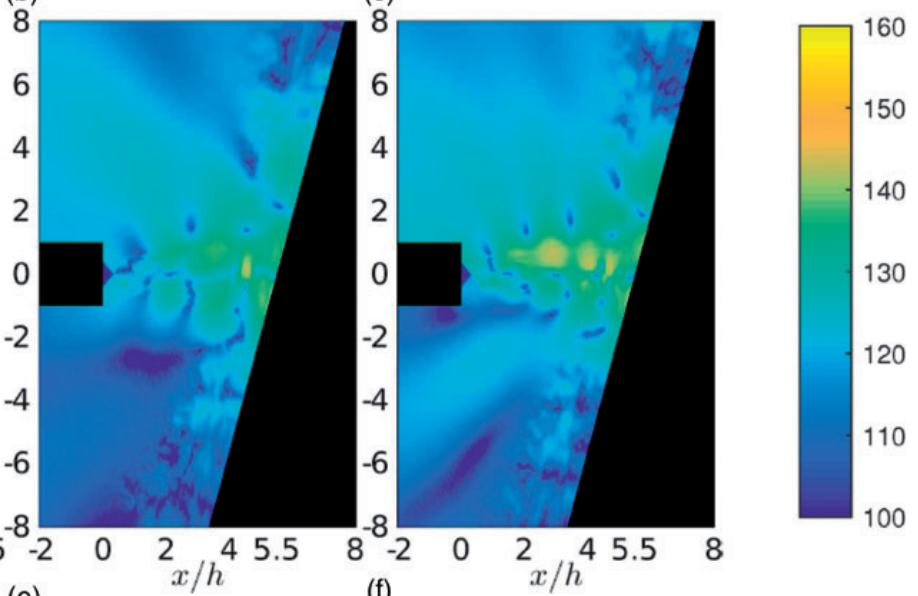

(d)

(e)

(f)
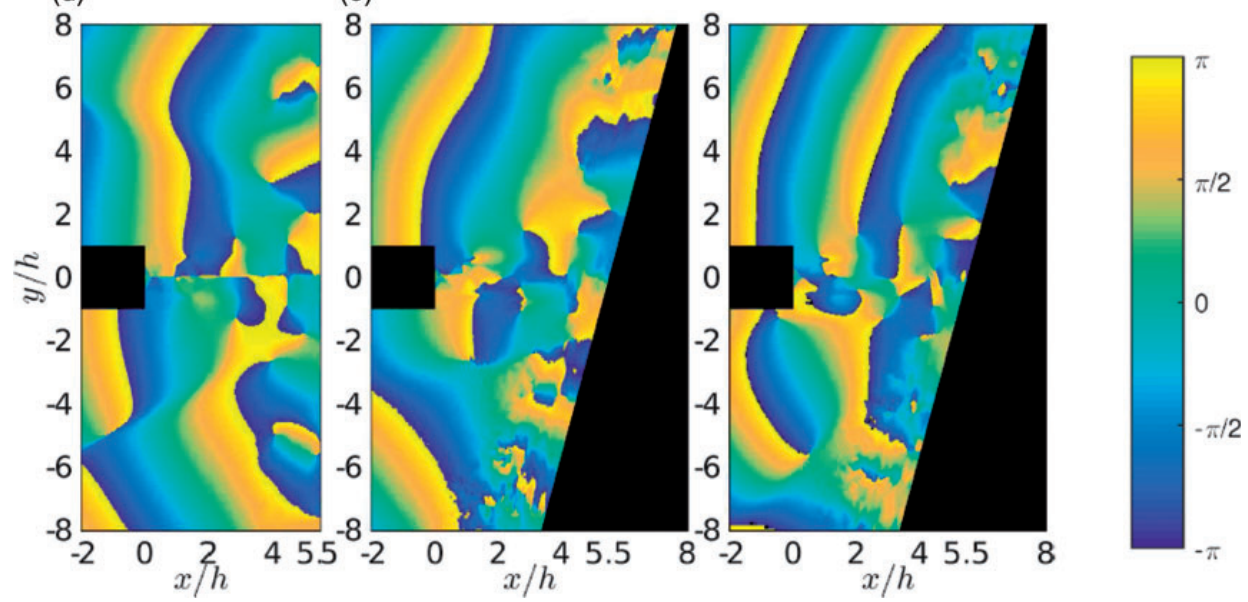

Figure 10. Amplitude (top) and phase (bottom) obtained for the pressure fields for (a and d) JetL5-90d at $S t_{2}=0.19$, (b and e) JetL5-75d at $S t_{1}=0.19$, and (c and f) JetL5-75d at $S t_{2}=0.25$. The nozzle and the plate are in black. The color ranges range over $60 \mathrm{~dB}$ for the amplitude fields and from $-\pi$ to $\pi$ for the phase fields.

the lower shear layer. This suggests that the feedback mechanism establishes mainly in the upper jet shear layer for JetL5-75d. This observation is consistent with the switch from the third mode to the fourth mode reported in the previous section. The nozzle-to-plate distance along the upper jet shear layer $L_{\text {upper }}$ increases from $5.5 \mathrm{~h}$ for JetL5-90d to $5.63 \mathrm{~h}$ for JetL5-75d. Therefore, it is not surprising that the same mode jump has been observed experimentally when the nozzle-to-plate distance is increased from $5.5 \mathrm{~h}$ to $5.85 \mathrm{~h}$ for normal impinging jets.

Those results are in agreement with the combination of models proposed in Gojon et al. ${ }^{17}$ Indeed, for this jet configuration, the tone Strouhal number $S t=0.19$ and the antisymmetric oscillation mode of the jet at this frequency are predicted. The tone at $S t=0.25$ is also 

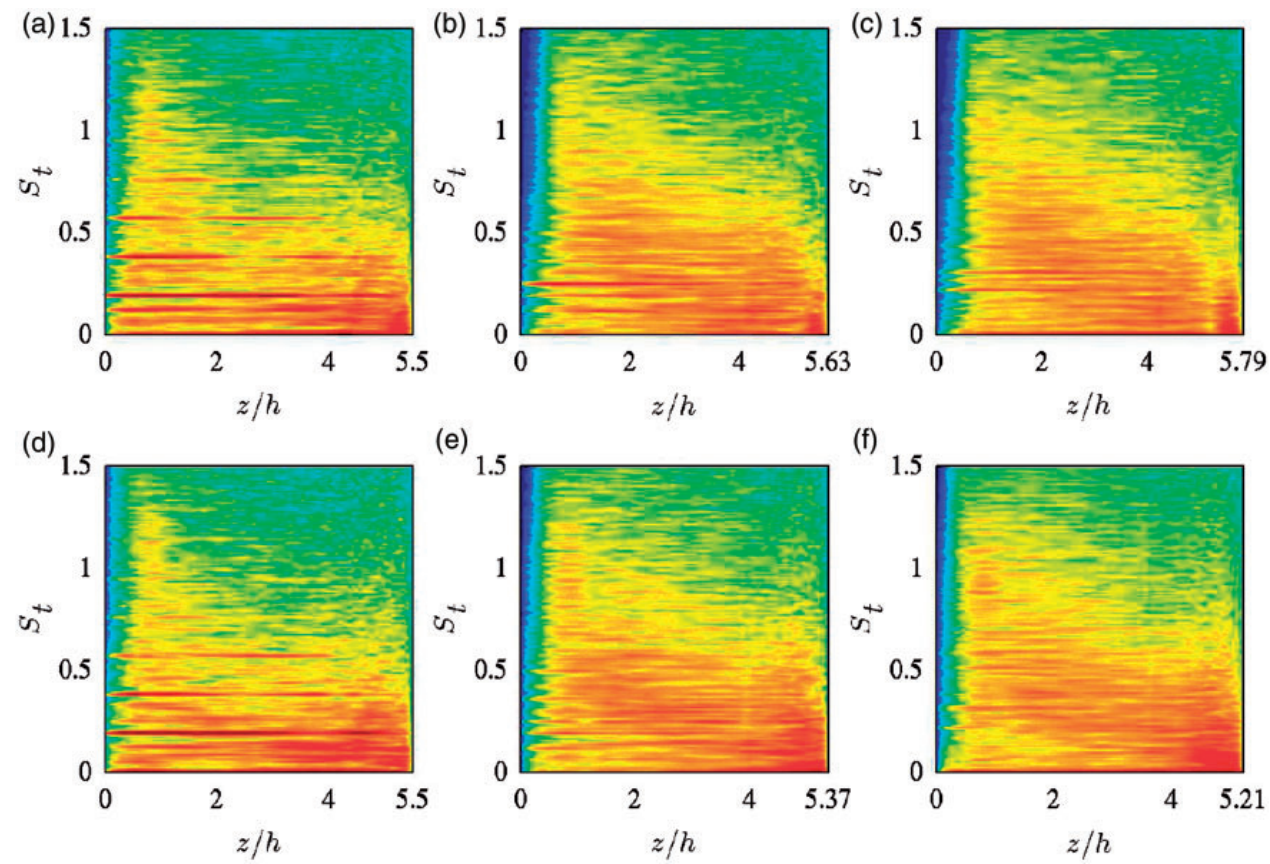

Figure II. Power spectral densities of axial velocity fluctuations $u_{z}{ }^{\prime}$ obtained along the (a to c) upper jet shear layer and ( $d$ to $f$ ) lower jet shear layer as functions of the Strouhal number and the axial coordinate for (a and d) JetL5-90d, (b and e) JetL5-75d, and ( $c$ and f) JetL5-60d. The color scale ranges over $50 \mathrm{~dB}$ from blue to red.

predicted with an antisymmetric oscillation mode of the jet. This oscillation mode is not clearly recovered in the phase field in Figure 10(c). It is probably due to the fact that almost only the upper jet shear layer maintains the aeroacoustic feedback mechanism, with phase contours that all seem to come from the upper jet shear layer region of impact in Figure 10(c), even in the region where $y<0$.

\section{Velocity spectra}

The spectra of axial velocity fluctuations calculated in the lower and upper jet shear layers are presented in Figure 11 as functions of the Strouhal number and the axial distance. They are computed using the Welch method with a $50 \%$ overlapping in time on one point in the spanwise direction. For JetL5-90d, a very similar behavior can be noted on both sides of the jet in agreement with the symmetry. A dominant tone at $S t=0.19$ is visible from the nozzle exit to the plate. Its first three harmonics are also seen, mainly near the nozzle exit. For JetL5-75d, different results are obtained in the two jet shear layers, as expected. In the lower shear layer, in Figure 11(e), a low-amplitude tone at $S t=0.19$ only appears near the nozzle exit, whereas in the upper shear layer, in Figure 11(b), a dominant tone at $S t=0.25$ and a secondary tone at $S t=0.19$ emerge. This result is consistent with the observation made in the previous section that the feedback mechanism establishes mainly in the upper jet shear layer in this case. For JetL5-60d, several tones of low amplitude can be identified around $S t=0.25$ in the upper shear layer, but they are less marked in the lower shear layer. 

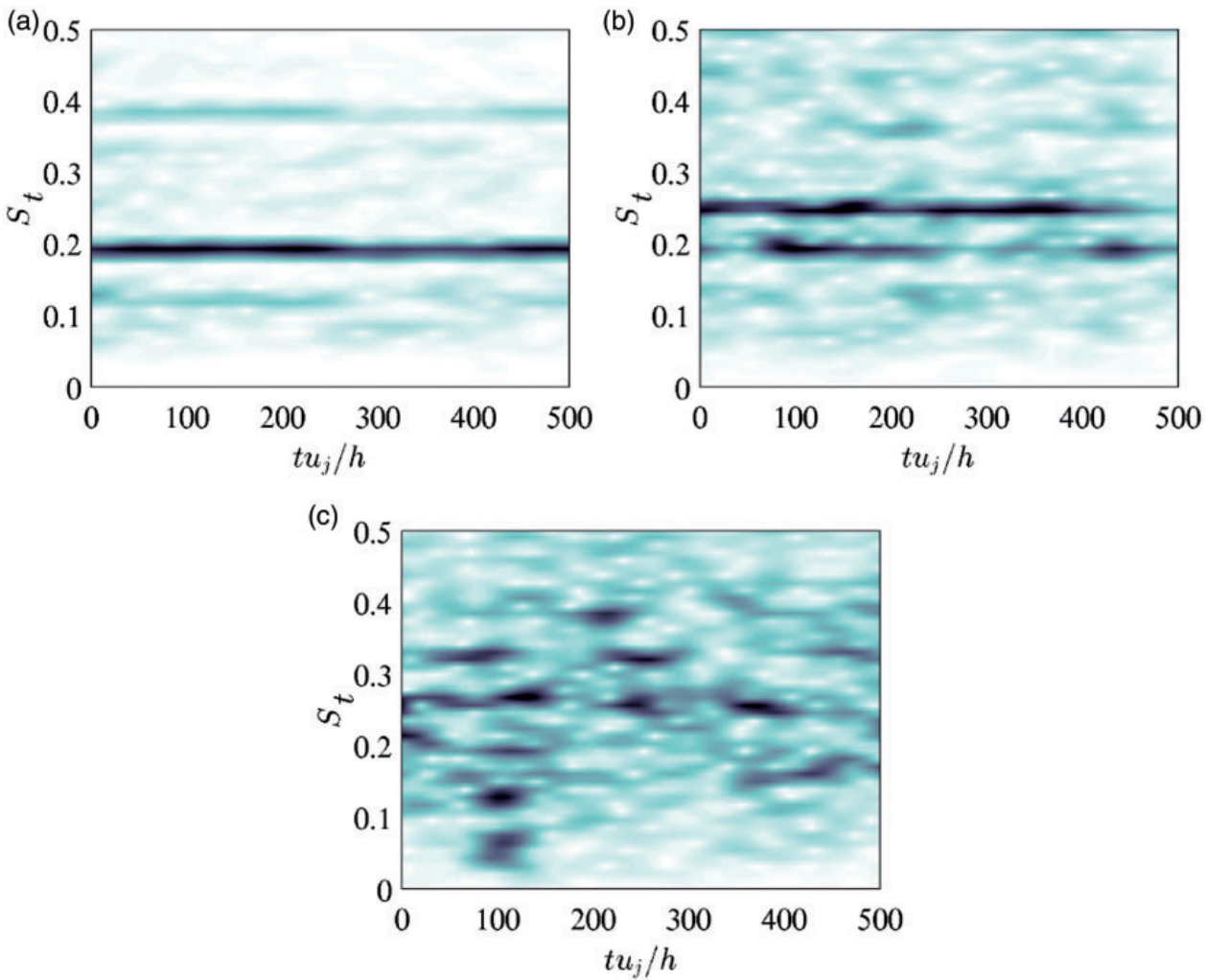

Figure 12. Sound pressure levels obtained at $x=0$ and $y=1.5 \mathrm{~h}$ as functions of time and Strouhal number for (a) JetL5-90d, (b) JetL5-75d, and (c) JetL5-60d. The color scales have been chosen arbitrarily and range over $30 \mathrm{~dB}$ for JetL5-90d, $15 \mathrm{~dB}$ for JetL5-75d, and $10 \mathrm{~dB}$ for JetL5-60d.

\section{Tone intermittency}

In order to determine whether the jets produce alternatively or simultaneously the different tones emerging in the spectra of Figure 7, a Fourier decomposition is applied to the pressure signal at $x=0$ and $y=1.5 h$ using a sliding window in time of size $35 u_{j} / h$. The results for the three jets are displayed in Figure 12 as functions of time and Strouhal number.

For JetL5-90d, in Figure 12(a), the contribution of the dominant tone frequency at $S t_{2}=$ 0.19 is clearly visible and coexists in time with those of its harmonic at $S t_{3}=0.38$ and of the secondary tone frequency at $S t_{1}=0.12$. For JetL5-75d, in Figure 12(b), the tone at $S t_{1}=0.19$ and a second tone at $S t_{2}=0.25$ appear simultaneously. Finally, for JetL5-60d, in Figure 12 (c), the tone at $S t=0.25$ can still be noticed and varies over time. Besides, several other tones, at $S t=0.08,0.13,0.19,0.32$, and 0.385 , stand out over short periods of time of about $50 u_{j} / h$. These tones can be linked to the modes $N=1,2,3,5$, and 6 of the aeroacoustic feedback mechanism. Thus, for an angle of impact of $60^{\circ}$, it seems that none of the modes of the feedback persists in time, but that several modes randomly establish during short periods of time. These rapid switches between different modes lead to several tones that are less energetic on average and centered on $S t=0.25$ for JetL5-60d in Figure 7(c). 


\section{Conclusion}

In this paper, the hydrodynamic and acoustic properties of three planar supersonic jets computed by compressible LESs are presented. The jets are ideally expanded, and have a Mach number $\mathcal{M}_{j}$ of 1.28 , and a Reynolds number $R e_{j}$ of $5 \times 10^{4}$. One jet impinging on a flat plate normally, and two other impinging jets with angles of impact of $60^{\circ}$ and $75^{\circ}$ are considered. Mean velocity flows and snapshots of density, fluctuating pressure, and vorticity are described. The mean convection velocity of the turbulent structures in the jet shear layers is determined. The results are consistent with theoretical models and with experimental values. The near pressure fields are then detailed. Several tones emerge in the pressure spectra in the vicinity of the nozzle. The frequencies associated with these tones compare well with measurements and with the classical model of the feedback mechanism. Besides, a jump from the third to the fourth mode and a reduction in intensity are observed, as the angle of impact deviates from $90^{\circ}$ to $75^{\circ}$. A reduction in the amplitude of the acoustic waves coming from the region of jet impact is also noticed. A Fourier decomposition is then applied to the near pressure fields in the $(x, y)$ plane. Hydrodynamic-acoustic standing waves are found in the amplitude fields of some tone frequencies. Moreover, for an angle of impact of $75^{\circ}$, higher amplitudes are found in the upper shear layer than in the lower shear layer, indicating that the feedback mechanisms establish mainly along the lip that is farther away from the plate when the impact angle is not normal. Velocity spectra in the jet shear layers also support this result. Indeed, the dominant tone frequency is only visible in the velocity spectra of the upper jet shear layer for an angle of impact of $75^{\circ}$. This observation is consistent with the jump observed from the third mode to the fourth mode. In this case, the nozzle-to-plate distance along the upper jet shear layer increases from $5.5 \mathrm{~h}$ for JetL5-90d to $5.63 h$ for JetL5-75d. Therefore, it seems natural that the present jump is similar that observed experimentally when the nozzle-to-plate distance is increased from $5.5 \mathrm{~h}$ to $5.85 h$ for normal impinging jets. Finally, using a Fourier decomposition over a sliding window in time, it is observed, for an angle of impact of $60^{\circ}$, that none of the modes of the feedback persists in time, but that several modes randomly establish during short periods of time. These rapid switches between different modes lead to several tones that are less energetic on average and centered on $S t=0.25$ for this impact angle.

\section{Declaration of conflicting interests}

The author(s) declared no potential conflicts of interest with respect to the research, authorship, and/or publication of this article.

\section{Funding}

The author(s) disclosed receipt of the following financial support for the research, authorship, and/or publication of this article: This work was performed using HPC resources of Pôle de Calcul Hautes Performances Dédiés and CNRS on Turing, Occigen (GENCI-IDRIS grant x2016a7178 and GENCICINES grant A0022A07178) and Eos (CALMIP, grant 2017-p1425).

\section{References}

1. Powell A. On edge tones and associated phenomena. Acta Acust United Acust 1953; 3: 233-243.

2. Ho C and Nosseir N. Dynamics of an impinging jet. Part 1. The feedback phenomenon. J Fluid Mech 1981; 105: 119-142. 
3. Nosseir N and Ho C. Dynamics of an impinging jet. Part 2. The noise generation. $J$ Fluid Mech 1982; 116: 379-391.

4. Henderson B and Powell A. Experiments concerning tones produced by an axisymmetric choked jet impinging on flat plates. J Sound Vib 1993; 168: 307-326.

5. Krothapalli A, Rajkuperan E, Alvi F, et al. Flow field and noise characteristics of a supersonic impinging jet. J Fluid Mech 1999; 392: 155-181.

6. Risborg A and Soria J. High-speed optical measurements of an underexpanded supersonic jet impinging on an inclined plate. In: 28th international congress on high-speed imaging and photonics, Canberra, Australia, 10 February 2009, paper no. 7126(F).

7. Buchmann N, Mitchell D, Ingvorsen K, et al. High spatial resolution imaging of a supersonic underexpanded jet impinging on a flat plate. In: Abstract from 6th Australian conference on laser diagnostics in fluid mechanics and combustion, Canberra, Australia, 5-7 December 2011.

8. Weightman J, Amili O, Honnery D, et al. An explanation for the phase lag in supersonic jet impingement. J Fluid Mech 2017; 815: R11-R111.

9. Henderson B, Bridges J and Wernet M. An experimental study of the oscillatory flow structure of tone-producing supersonic impinging jets. J Fluid Mech 2005; 542: 115-137.

10. Gojon R and Bogey C. Flow structure oscillations and tone production in underexpanded impinging round jets. AIAA $J$ 2017; 55: 1792-1805.

11. Bogey $\mathrm{C}$ and Gojon R. Feedback loop and upwind-propagating waves in ideally-expanded supersonic impinging round jets. J Fluid Mech 2017; 823: 562-591.

12. Gojon R and Bogey C. Flow features near plate impinged by ideally expanded and underexpanded round jets. AIAA J 2018; 56: 445-457.

13. Arthurs D and Ziada S. Self-excited oscillations of a high-speed impinging planar jet. $J$ Fluids Struct 2012; 34: 236-258.

14. Norum T. Supersonic rectangular jet impingement noise experiments. AIAA J 1991; 29: $1051-1057$.

15. Tam $\mathrm{C}$ and Norum $\mathrm{T}$. Impingement tones of large aspect ratio supersonic rectangular jets. AIAA $J$ 1992; 30: 304-311.

16. Hourigan K, Rudman $\mathrm{M}$ and Brocher E. The feedback loop in impinging two-dimensional highsubsonic and supersonic jets. Exp Therm Fluid Sci 1996; 12: 265-270.

17. Gojon R, Bogey C and Marsden O. Investigation of tone generation in ideally expanded supersonic planar impinging jets using large-eddy simulation. J Fluid Mech 2016; 808: 90-115.

18. Gojon R, Bogey $\mathrm{C}$ and Mihaescu M. Oscillation modes in screeching jets. AIAA J 2018; 56: $2918-2924$.

19. Nonomura T, Tsutsumi S, Takaki R, et al. Impact of spatial and temporal resolution on the aeroacoustic wave from a two-dimensional impinging jet. In: 7 th international conference on computational fluid dynamics, Big Island, Hawaii, 9-13 July 2012, paper no. ICCFD7-3103.

20. Nonomura T, Goto Y and Fujii K. Aeroacoustic waves generated from a supersonic jet impinging on an inclined flat plate. Int J Aeroacoust 2011; 10: 401-425.

21. Tsutsumi S, Nonomura T, Fujii K, et al. Analysis of acoustic wave from supersonic jets impinging to an inclined flat plate. In: 7th international conference on computational fluid dynamics, Big Island, Hawaii, 9-13 July 2012, paper no. ICCFD7-3104.

22. Thurow B, Samimy M and Lempert W. Structure of a supersonic impinging rectangular jet via real-time optical diagnostics. In: 32nd AIAA fluid dynamics conference, St. Louis, MO, 24-26 June 2002, AIAA paper no. 2002-2865.

23. Bogey C and Bailly C. A family of low dispersive and low dissipative explicit schemes for flow and noise computations. J Comput Phys 2004; 194: 194-214.

24. Berland J, Bogey C, Marsden O, et al. High-order, low dispersive and low dissipative explicit schemes for multiple-scale and boundary problems. J Comput Phys 2007; 224: 637-662.

25. Tam $\mathrm{C}$ and Dong Z. Wall boundary conditions for high-order finite-difference schemes in computational aeroacoustics. Theor Comput Fluid Dyn 1994; 6: 303-322. 
26. Bogey C, Marsden O and Bailly C. Large-eddy simulation of the flow and acoustic fields of a Reynolds number $10^{5}$ subsonic jet with tripped exit boundary layers. Phys Fluids 2011; 23: 035104.

27. Bogey C, Marsden O and Bailly C. Influence of initial turbulence level on the flow and sound fields of a subsonic jet at a diameter-based Reynolds number of $10^{5}$. J Fluid Mech 2012; 701: 352-385.

28. Bogey C, de Cacqueray $\mathrm{N}$ and Bailly C. A shock-capturing methodology based on adaptative spatial filtering for high-order non-linear computations. J Comput Phys 2009; 228: 1447-1465.

29. de Cacqueray N, Bogey $\mathrm{C}$ and Bailly $\mathrm{C}$. Investigation of a high-Mach-number overexpanded jet using large-eddy simulation. AIAA J 2011; 49: 2171-2182.

30. Gojon R and Bogey C. Numerical study of the flow and the near acoustic fields of an underexpanded round free jet generating two screech tones. Int J Aeroacoust 2017; 16: 603-625.

31. Tam $\mathrm{C}$ and $\mathrm{Hu} \mathrm{F}$. An optimized multi-dimensional interpolation scheme for computational aeroacoustics applications using overset grids. In: 10th AIAA/CEAS aeroacoustics conference, $10-12$ May 2004, Manchester, UK; AIAA paper no. 2004-2812.

32. Chicheportiche $\mathrm{J}$ and Gloerfelt $\mathrm{X}$. Techniques de recouvrement de maillages pour le calcul direct en aéroacoustique, 2011.

33. Marsden $\mathrm{O}$, Bogey $\mathrm{C}$ and Bailly C. High-order curvilinear simulations of flows around nonCartesian bodies. J Comp Acoust 2005; 13: 731-748.

34. Kremer F and Bogey C. Large-eddy simulation of turbulent channel flow using relaxation filtering: resolution requirement and Reynolds number effects. Comput Fluids 2015; 116: 17-28.

35. Viazzo S, Dejoan A and Schiestel R. Spectral features of the wall-pressure fluctuations in turbulent wall flows with and without perturbations using les. Int J Heat Fluid Flow 2001; 22: 39-52.

36. Gloerfelt X and Berland J. Direct computation of turbulent boundary layer noise. In: 15th AIAA/ CEAS aeroacoustics conference, Miami, FL, 11-13 May 2009, AIAA paper no. 2009-3401.

37. Schlatter P, Li Q, Brethouwer G, et al. Simulations of spatially evolving turbulent boundary layers up to $\mathrm{Re}_{\theta}=4300$. Int $J$ Heat Fluid Flow 2010; 31: 251-261.

38. Irwin H. Measurements in a self-preserving plane wall jet in a positive pressure gradient. $J$ Fluid Mech 1973; 61: 33-63.

39. George W, Abrahamsson H, Eriksson J, et al. A similarity theory for the turbulent plane wall jet without external stream. J Fluid Mech 2000; 425: 367-411.

40. Panda J, Raman $\mathrm{G}$ and Zaman $\mathrm{K}$. Underexpanded screeching jets from circular, rectangular and elliptic nozzles. In: 3th AIAA/CEAS aeroacoustics conference, Atlanta, GA, 12 - 14 May 1997, AIAA paper no. 97-1623.

41. Papamoschou D and Roshko A. The compressible turbulent shear layer: an experimental study. J Fluid Mech 1988; 197: 453-477.

42. de Cacqueray $\mathrm{N}$ and Bogey $\mathrm{C}$. Noise of an overexpanded Mach 3.3 jet: non-linear propagation effects and correlations with flow. Int J Aeroacoust 2014; 13: 607-632.

43. Ffowcs-Williams J, Simson J and Virchis V. 'Crackle': an annoying component of jet noise. J Fluid Mech 1975; 71: 251-271. 